\title{
Sustracción Radiográfica Digital de la Estructura Dentaria Removida Durante el Acceso Endodóntico en Incisivos Mandibulares
}

\author{
Digital Subtraction Radiography of Dental Hard Tissue Removed \\ During Endodontic Access in Mandibular Incisors
}

\begin{abstract}
María Belén Ibarra-Benítez; Alicia Elizabeth Caballero-Contrera; Luis Fernando Páez-Reyes; Vicente R. Fretes \& Carlos G. Adorno
\end{abstract}

IBARRA-BENÍTEZ, M. B.; CABALLERO-CONTRERA, A. E.; PÁEZ-REYES, L. F.; FRETES, V. R. \& ADORNO, C. G. Sustracción radiográfica digital de la estructura dentaria removida durante el acceso endodóntico en incisivos mandibulares. Int. J. Odontostomat., 15(3):653-659, 2021.

RESUMEN: El objetivo de este estudio fue comparar la estructura dentaria removida utilizando dos técnicas de acceso endodóntico diferentes en incisivos mandibulares. Treinta y cinco incisivos mandibulares extraídos fueron pesados individualmente con una balanza de precisión. Después de las radiografías iniciales tomadas por vestibular y proximal, los dientes fueron codificados y asignados aleatoriamente a dos grupos: acceso convencional $(n=17)$ o acceso incisal $(n=18)$. Luego se volvieron a pesar los dientes y se repitieron las radiografías. Las radiografías digitales se cargaron a un editor de imágenes (Adobe Photoshop) para la sustracción digital de las imágenes por las incidencias VP y MD. Dos observadores independientes y calibrados realizaron las mediciones de las áreas sustraídas. La concordancia inter-observador fue casi perfecta; coeficiente de correlación intraclase igual a 0,94 y 0,98 para las incidencias MD y VP, respectivamente. La prueba de Mann-Whitney encontró diferencias estadísticamente significativas entre los tipos de accesos en cuanto a la diferencia mediana de los pesos $(p=0,0073)$ y el área $V L(p=0.0023)$. También encontró una diferencia estadísticamente significativa entre los tipos de acceso en cuanto al área MD (Prueba t de Welch, $p<0,001$ ). La diferencia de peso fue menor para el acceso incisal que para el acceso convencional. El acceso incisal removió menos tejido dentario que el acceso convencional.

PALABRAS CLAVE: endodoncia, tratamiento del conducto radicular, técnica de sustracción.

\section{INTRODUCCIÓN}

El acceso endodóntico es el primer paso de una serie de procedimientos del tratamiento de endodoncia y si es correctamente realizado, favorece la ejecución de las demás fases del tratamiento (Krapez \& Fidler, 2013). Un acceso correcto promueve la localización de los conductos, permite un acceso directo a los mismos y conserva estructura dentaria (Varghese et al., 2016). Indudablemente, antes de realizar el acceso es importante conocer la anatomía externa e interna del diente a tratar.

Los incisivos mandibulares son los dientes más pequeños del adulto humano. Poseen una raíz amplia en sentido vestíbulo-lingual y estrecha en sentido mesiodistal, pudiendo presentar un conducto de forma ovoide o acintado a la sección transversal. Si bien existe una gran variación reportada en la prevalencia de más de un conducto, recientemente un estudio utilizando microtomografía computarizada reportó que la misma fue de $50 \%$ y $38 \%$ para incisivos centrales y laterales, respectivamente (Leoni et al., 2014).

La forma del acceso esta relacionada con la morfología de la cámara pulpar del diente a tratar. Al retirar el techo cameral, la proyección coronal de las paredes laterales de la cámara pulpar localiza al acceso sobre el borde incisal. Sin embargo, el acceso en incisivos mandibulares se realiza tradicionalmente por la 
cara lingual por razones estéticas y restauradoras (Mauger et al., 1999). El punto de acceso es justo por encima del cíngulo, y se extiende en sentido vestíbulo-lingual adquiriendo finalmente una forma ovoide o triangular con base incisal. Este diseño puede resultar en un aumento de estructura dentaria removida que podría comprometer la integridad estructural del diente (Reeh et al., 1989) debido a que la cantidad de dentina remanente esta directamente relacionada a la resistencia a la fractura del diente (Lertchirakarn et al., 2003). La preparación del acceso endodóntico requiere de un equilibrio preciso entre proporcionar un acceso adecuado para la terapia endodóntica y la preservación de la estructura dental, factor importante para la restauración y supervivencia a largo plazo del diente. Es por eso que en la actualidad, con el desarrollo de nuevas técnicas y materiales, tanto para la realización del tratamiento endodóntico como para la restauración final, surgieron alternativas al diseño convencional del acceso coronario en incisivos mandibulares (Logani et al., 2009).

El acceso a través del borde incisal proporcionaría un acceso en línea recta al tercio apical de la raíz, reduciendo el transporte apical producido durante la instrumentación (Yahata et al., 2017), preservando la dentina en el área pericervical (Varghese et al.), conservando mayor estructura dentaria y permitiendo una mejor preparación biomecánica, especialmente en el tercio apical del conducto radicular (Mauger et al.). Todo procedimiento y técnica que respeta la salud, función y estética de los tejidos orales por medio de la prevención de la ocurrencia de la enfermedad o la interrupción de su progreso con mínima cantidad de pérdida de tejido puede considerarse como odontología mínimamente invasiva (Novy \& Fuller, 2008) y el acceso a través del borde incisal en incisivos mandibulares podría considerarse como una aplicación de estos conceptos.

La técnica de sustracción digital es una herramienta valiosa para detectar pequeños cambios entre dos radiografías. Ha sido utilizada para estudiar el proceso de reparación ósea de la periodontitis apical crónica luego del tratamiento de conducto (Carvalho et al., 2007; Benfica e Silva et al., 2010), detección de fracturas verticales (Queiroz et al., 2016), diagnóstico de reabsorciones cervicales externas (Sunku et al., 2011), identificación de las características anatómicas de los sistemas de conductos en "C" (Fan et al., 2008), evaluación del transporte apical producido por la instrumentación rotatoria (Kunert et al., 2010), entre otras. Esta técnica detecta cambios de radioopacidades entre dos radiografías tomadas en diferentes momen- tos, antes y después de un evento determinado, resaltando las diferencias entre ambas imágenes (Queiroz et al.).

El acceso endodóntico bien realizado proporciona la posibilidad de ejecución correcta de las demás etapas del tratamiento y facilita la localización y exploración de los conductos radiculares. Realizar el acceso en función de la anatomía del sistema de conductos radiculares es de fundamental importancia para evitar la posibilidad de escalones y evitar la tensión excesiva en los instrumentos endodónticos. La hipótesis nula del presente trabajo fue que no hay diferencia en el tipo de acceso endodóntico en cuanto al área de estructura dentaria removida. El objetivo de trabajo fue comparar la estructura dentaria removida utilizando dos técnicas de acceso endodóntico diferentes en incisivos mandibulares, por medio de la sustracción digital.

\section{MATERIAL Y MÉTODO}

El presente estudio fue experimental con muestreo no probabilístico por conveniencia. Se utilizaron incisivos centrales y laterales mandibulares extraídos por motivos periodontales. Luego de la examinación visual y por radiografías periapicales de perfil se seleccionaron dientes sin tratamiento endodóntico, con corona íntegra y donde la cámara pulpar era discernible. Los dientes que presentaban caries, fracturas, dientes con tratamiento endodóntico previos, erosiones y/o abfracciones fueron eliminados.

La estimación del tamaño de la muestra fue calculada con el software de G*Power 3.1.9.2, estimándose que 17 dientes por grupo serían necesarios para detectar un tamaño de efecto igual a 1 por medio de la prueba $t$ de muestras independientes con poder estadístico de $80 \%$ y con un error tipo I de 0,05 (a dos colas).

Los dientes seleccionados fueron limpiados con puntas de ultrasonido y luego almacenados en una solución de suero fisiológico hasta el momento de iniciar el procedimiento. Se confeccionaron troqueles de acrílico autocurado con $15 \mathrm{~mm}$ de alto y $22 \mathrm{~mm}$ de ancho y largo, en los cuales se montaron los dientes previamente enumerados.

Los dientes fueron pesados en una balanza digital y se registraron los valores pre-operatorios. Posteriormente se realizó la radiografía digital inicial de 
cada uno de ellos con ayuda de un sensor radiográfico intraoral (One T1, Owandy Radiology SAS®, CroissyBeaubourg, Francia). La posición de las radiografías fue estandarizada con ayuda de un posicionador. Las imágenes obtenidas fueron almacenaron las imágenes en formato tiff.

Los dientes fueron asignados aleatoriamente a uno de dos grupos:

Grupo 1: Acceso convencional. Se realizó el acceso a través de la superficie lingual, el punto de penetración inicial fue el centro exacto de la superficie lingual, justo por encima del cíngulo con la fresa perpendicular a la superficie. La penetración inicial de la cavidad se realizó con una fresa redonda nro. 1012 (FAVA, São Paulo, Brasil) operada a alta velocidad y con irrigación constante. El contorno de la cavidad de acceso fue de forma triangular con base orientada al borde incisal. Luego, con una fresa de punta inactiva (nro. 3080, FAVA) se alisaron las paredes vestibular y lingual y se eliminó el hombro lingual (Figs. 1a, 1b, 1d, 1e).

Grupo 2: Acceso Incisal. El acceso se realizó a través del borde incisal, el punto de penetración inicial fue el centro del borde incisal. La penetración inicial de la cavidad se preparó con una fresa redonda (nro. 1012 FAVA), operada alta velocidad y con irrigación constante. La fresa se ubicó en forma paralela al eje longitudinal del diente, resultando en un acceso de forma circular. La preparación se terminó con una fresa de punta inactiva (nro. 3080, FAVA) (Figs. 1g, 1h, 1j, 1k).

Todos los accesos se realizaron bajo magnificación utilizando un microscopio operatorio. Una vez terminados los accesos de ambos grupos se procedió a pesar cada diente y a anotar sus valores para luego tomar la radiografía final. Una vez realizadas todas las radiografías se procedió a superponer las imágenes de cada diente con el programa Adobe $₫$ Photoshop ${ }^{\circledR}$ CS6 Versión 13.0.01 para determi-
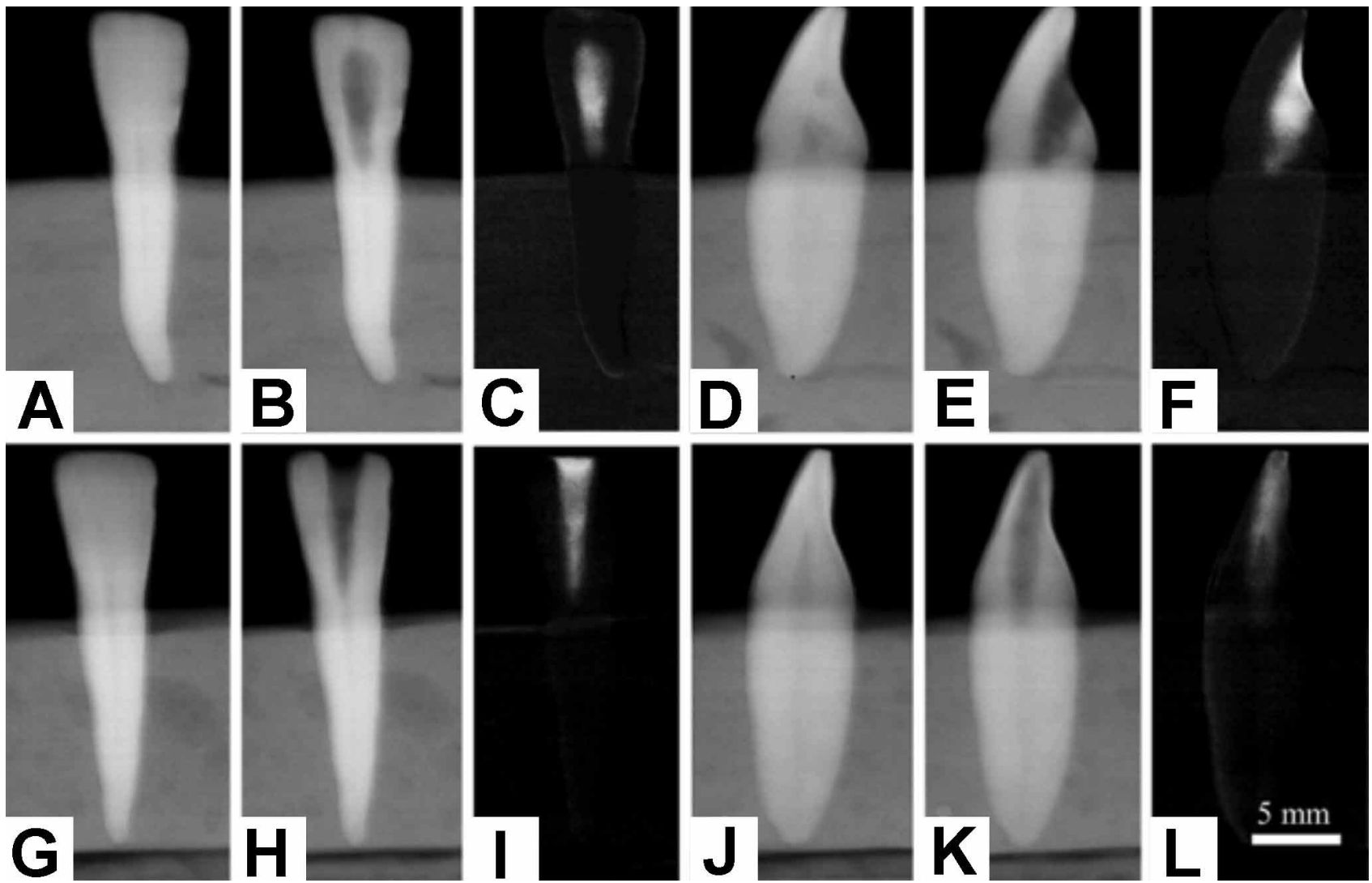

Fig. 1. Radiografías pre y post-operatorias. Acceso convencional: incidencia vestibular pre operatoria (a), vestibular post operatoria (b y sustracción radiográfica de ambas imágenes (c). Incidencia proximal pre operatoria (d), proximal post operatoria (e) y sustracción radiográfica de ambas imágenes (f). Acceso incisal: vestibular pre operatoria (g), vestibular post operatoria (h) y sustracción radiográfica de ambas imágenes (f). Incidencia proximal pre operatoria (g), proximal post opertaria (h) y sustracción radiográfica de ambas imágenes (I). 
nar el área de tejido dentario perdido en $\mathrm{mm}^{2}$ durante el acceso coronario. Mediante la sustracción radiográfica se pudo demarcar dicha área con exactitud (Figs. 1c, 1f, 1i, 11).

El área del acceso fue medida por dos evaluadores independientes calibrados para tener dos pares de mediciones por incidencia de rayo; MD y VL. Para evaluar la concordancia inter-observador en cada par de mediciones se calculó el coeficiente de correlación intraclase $(\mathrm{CCl})$ de dos factores, efectos mixtos de medidas únicas (modelo $\mathrm{CCl} 3,1$ ) (Shrout \& Fleiss, 1979) y sus respectivos intervalos de confianza. Para interpretar el $\mathrm{CCl}$ se utilizó la siguiente escala: pobre/ leve $(0,00-0,20)$, aceptable $(0,21-0,40)$, moderada $(0,41-0,60)$, considerable $(0,61-0,80)$ y casi perfecta $(0,81-1,00)$ (Landis \& Koch, 1977). Luego se promediaron las observaciones de ambos observadores resultando en las áreas promediadas por MD y por VL. Se halló la diferencia entre los pesos de los dientes antes y después de los accesos. Esta diferencia representaría el peso de la estructura dentaria removida.

La variable independiente, de escala de medición nominal, fue el tipo de acceso; convencional o incisal. Las variables diferencia de peso, área MD y área $V L$ fueron las variables dependientes medidas en escala continua. Los supuestos estadísticos de normalidad y homogeneidad de varianzas fueron evaluados por medio de las pruebas de Shapiro-Wilk y Levene, respectivamente. La prueba t de Student se utilizaría para determinar si había diferencias entre los grupos si se cumplían todos los supuestos estadísticos, de lo contrario se utilizaría la prueba U de Mann Whitney. Todos los análisis estadísticos fueron realizados con el paquete estadístico $R$ versión 3.3.1., fijándose el nivel de significancia en 0,05.

\section{RESULTADOS}

Las estadísticas descriptivas se resumen en la Tabla I. Los niveles de acuerdo interobservador fueron casi perfectos para las incidencias $\mathrm{MD}(\mathrm{CCl}=$
0,94, IC95\%: 0,89-0,97) y VL (CCI = 0,98, IC95\%: $0,95-0,99)$. En la figura 2 se observa un diagrama de cajas del área de acceso medida en las radiografías según incidencia y en la figura 3 un diagrama de puntos de la diferencia de peso del diente antes y después del acceso.

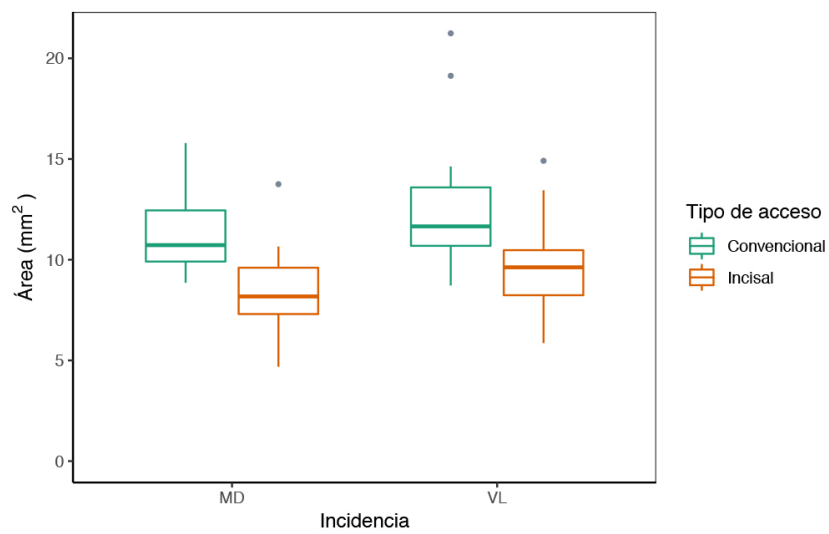

Fig. 2. Diagrama de cajas del área de acceso medida en las radiografías según incidencia (mesiodistal (MD) o vestíbulo lingual $(\mathrm{VL}))$. El acceso incisal produjo áreas significativamente menores que el acceso convencional en ambas incidencias. La línea más gruesa corresponde a la mediana.

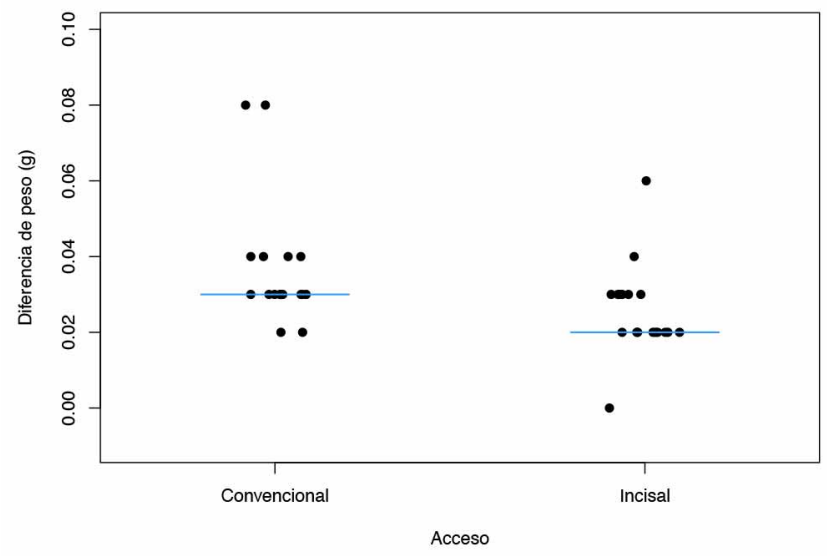

Fig. 3. Diagrama de puntos de la diferencia de peso del diente antes y después del acceso según el tipo de acceso. La diferencia de peso fue menor para el acceso incisal que para el acceso convencional con una diferencia estadísticamente significativa (Prueba U de Mann Whitney, $p=0,0073$ ). Las líneas horizontales azules representan la mediana.

Tabla I. Estadísticas descriptivas (Mediana y cuartiles 1 (Q1) y 2 (Q3) de la diferencia de peso y áreas de acceso medido por sustracción radiográfica en ambas incidencias según el tipo de acceso.

\begin{tabular}{lcccccc}
\hline \multirow{2}{*}{ Tipo de acceso } & \multicolumn{2}{c}{ Diferencia de peso $(\mathrm{g})$} & \multicolumn{2}{c}{ Área $\mathrm{MD}\left(\mathrm{mm}^{3}\right)$} & \multicolumn{2}{c}{ Área $\mathrm{VL}\left(\mathrm{mm}^{3}\right)$} \\
& Mediana & $\mathrm{Q}_{1}-\mathrm{Q}_{3}$ & Mediana & $\mathrm{Q}_{1}-\mathrm{Q}_{3}$ & Mediana & $\mathrm{Q}_{1}-\mathrm{Q}_{3}$ \\
\hline Convencional & 0,03 & $0,02-0,04$ & 10,36 & $9,86-12,39$ & 11,58 & $9,38-13,16$ \\
Incisal $(\mathrm{n}=17)$ & 0,02 & $0,02-0,03$ & 8,17 & $7,30-9,60$ & 9,62 & $8,24-10,47$ \\
\hline
\end{tabular}


Todos los datos de las variables dependientes presentaron homogeneidad de varianzas (Prueba de Levene, $p>0,05)$. La variable área MD tenía distribución normal (Shapiro-Wilk, $p=0,9025)$; pero los datos de la diferencia de peso y área VL no presentaron distribución normal (Shapiro-Wilk, $p<0,05$ ).

La prueba de Mann-Whitney encontró diferencias estadísticamente significativas entre los tipos de accesos en cuanto a la diferencia mediana de los pesos $(p=0,0073)$ y el área $V L(p=0.0023)$. También encontró una diferencia estadísticamente significativa entre los tipos de acceso en cuanto al área MD (Prueba t de Welch, $p<0,001$ ).

\section{DISCUSIÓN}

El presente estudio comparó, por medio de la sustracción digital, la estructura dentaria removida durante el acceso endodóntico en dientes incisivos mandibulares utilizando dos técnicas de acceso endodóntico, una convencional y otra incisal. Existe actualmente una tendencia a la práctica de procedimientos mínimamente invasivos, cuyo objetivo es conservar al máximo el tejido dental (Özyürek et al., 2018) y la aplicación de ese concepto se extendió también al acceso endodóntico. La técnica de acceso incisal es una aplicación de los conceptos de la odontología mínimamente invasiva que consisten básicamente en un "respeto sistemático por la estructura dentaria original” (Gutmann, 2013).

Para lograr un acceso endodóntico ideal, es necesario tener en mente la anatomía interna, como el tamaño y forma de la cámara pulpar, la cantidad de conductos radiculares, su posición y curvatura (Logani et al.). Es sabido que el acceso endodóntico correcto permite una mejor localización, medición, limpieza, modelado y obturación del conducto. Correctamente realizado puede prevenir complicaciones iatrogénicas como escalones y transporte apical. Es por esto que varios estudios mideron el impacto que pudiera tener los accesos conservadores en diferentes dientes sobre el resto de los procedimientos endodónticos; como la localización de los conductos (Rover et al., 2017), instrumentación (Varghese et al.; Rover et al., 2017; Yahata et al.; Alovisi et al., 2018; Rover et. al., 2020), resistencia a la fractura (Chlup et al., 2017; Plotino et al., 2017; Rover et al., 2017; Sabeti et al., 2018; Özyürek et al.; Roperto et al., 2019; Rover et. al., 2020) y la remoción de guttapercha durante el retratamiento (Fatima et al., 2018). Los molares, seguido por los premolares, son los dientes que más han sido estudiados en cuanto al impacto del acceso mínimamente invasivo. Debido a que los grupos dentarios difieren morfológicamente, es preciso evaluar el impacto clínico de los conceptos de la odontología mínimamente invasiva sobre los diferentes pasos de la terapia endodóntica. Por ejemplo, el acceso mínimamente invasivo permitió mayor conservación de dentina coronaria en incisivos centrales superiores, segundos premolares mandibulares y primeros molares mandibulares, pero solo tuvieron una mayor resistencia a la fractura los premolares y molares, no así los incisivos (Krishan et al., 2014). Cabe resaltar que estos dientes fueron sometidos a la prueba de resistencia a la fractura sin la restauración coronaria, a diferencia de una situación clínica en que se busca restaurar los dientes antes de someterlos a las fuerzas oclusales. En cambio, D'amico et al. (2019) reportaron que no hubo diferencias estadísticamente significativas en la resistencia a la fractura en incisivos mandibulares tratados endodónticamente y con acceso realizados de forma convencional o conservadora (bucal o lingual) y restaurados con resina composite. Esto fue corroborado también por un estudio reciente (Roperto et al.), quienes reportaron que independientemente del diseño de la cavidad de acceso, las cavidades que preservaban el reborde marginal no afectaron la resistencia a la fractura, modo de fallo o distribución de las tensiones en dientes premolares restaurados con resina compuesta; los dientes tratados endodónticamente y restaurados con resina exhibieron un comportamiento biomecánico similar al de un diente intacto. Similarmente, en incisivos mandibulares no se encontraron diferencias entre el acceso convencional a través de la cara lingual y el acceso conservador a través del borde incisal (Rover et. al., 2020)

En el presente estudio se observó que el acceso incisal logró una menor remoción de estructura dentaria (Tabla I). En los incisivos anteriores en general la dirección de la cavidad de acceso convencional es diferente a la dirección del conducto radicular, especialmente en su tercio apical lo que dificulta la realización de un tratamiento sin interferencias. Por tanto, se puede lograr un acceso en línea recta a través del borde incisal o la superficie labial (Krapez \& Fidler). En consecuencia, un estudio reciente (Yahata et al.) reportó que el acceso incisal en réplicas tridimensionales de acrílico permitía un mejor centrado de la lima durante la instrumentación, con un menor transporte apical en comparación con el acceso tradicional. Al permitir el centrado de la lima durante la instrumentación, se facilita el acceso a todas las áreas 
críticas del conducto, y por tanto una conformación y limpieza más efectiva. Una lima no centrada desgastará a las paredes del conducto de una manera desigual potencialmente produciendo transporte apical. Efectivamente, mediante la tomografía computarizada se comparó la influencia de las cavidades de acceso convencional e incisal en el desgaste de la dentina peri-cervical en los dientes anteriores mandibulares (Varghese et al.). Los autores observaron que el desgaste de las paredes durante la instrumentación era más parejo para el acceso incisal y que hubo un excesivo desgaste de la pared lingual durante el acceso convencional.

Logani et al., compararon el acceso lingual convencional con una preparación de acceso labial en los dientes anteriores mandibulares, con la finalidad de lograr la máxima conservación de la estructura dental coronal pesando el diente antes y después de la preparación de la cavidad de acceso; se observó que el tejido dental perdido durante el acceso lingual fue significativamente mayor al que se pierde durante la preparación de acceso labial. Este hallazgo esta en concordancia con los resultados del presente estudio, en que se observó una diferencia de $0,01 \mathrm{~g}$ entre los pesos medianos de los dientes después del acceso, con el acceso incisal removiendo menor cantidad de estructura dentaria.

Las mediciones cuantitativas por medio de la sustracción radiográfica digital poseen una mayor fiabilidad en comparación con radiografías convencionales. Sin embargo, la técnica depende de la reproducibilidad de la imagen radiográfica en cuanto al posicionamiento (distancias foco-objeto- sensor) y configuración del disparo (total de radiación), para evitar distorsiones geométricas y minimizar diferencias de densidad y contraste (Carvalho et al.; Benfica e Silva et al.). En el presente estudio se utilizó un posicionador similar al utilizado por lqbal et al.. (2003) y se utilizó el mismo aparato de rayo bajo la misma configuración. Esto permitió estandarizar la distancia foco-objeto-sensor en todas las radiografías, lo que favoreció la superposición de las imágenes para la sustracción digital.

Dentro de las limitaciones del presente estudio se puede concluir que el acceso convencional en incisivos mandibulares implica una mayor remoción de estructura dentaria en comparación al acceso incisal que se mostró más conservador en cuanto a las mediciones realizadas de peso y área. Se rechaza la hipótesis nula de que no hay diferencia en el tipo de acceso cavitario en cuanto al área de estructura dentaria removida.
IBARRA-BENITTEZ, M. B.; CABALLERO-CONTRERA, A. E.; PÁEZ-REYES, L. F.; FRETES, V. R. \& ADORNO, C. G. Digital subtraction radiography of dental hard tissue removed during endodontic access in mandibular incisors. Int. J. Odontostomat., 15(3):653-659, 2021.

ABSTRACT: The aim of this study was to compare the area of tooth structure removed during two different endodontic access techniques in lower incisors. Thirty-two extracted lower incisors were weighed individually on a precision scale. After initial digital radiographs were taken from buccal and proximal, the teeth were coded and randomly assigned to two different groups: conventional access and incisal access. Once this stage was completed, each tooth was weighed again and 2 postoperative radiographs was taken again, buccal and proximal. Using an image processing software and digital subtraction technique, 2 independent and calibrated evaluators measured difference in tooth structure after access procedures. Interobserver agreement was almost perfect: intraclass correlation coefficient equal to 0,94 and 0,98 for proximal and buccal incidences, respectively. The Mann-Whitney test found statistically significant differences between the types of accesses in terms of weight difference $(p=0.0073)$ and area $V L(p=0.0023)$. Additionally, a statistically significant difference between the types of access in the MD area (Welch's t test, $p<0.005$ ) was observed. The difference in weight was less for incisal access than for conventional access with a statistically significant difference, in terms of MD and VL area, incisal access and areas of smaller size than conventional access.

KEY WORDS: endodontics, root canal therapy, subtraction technique.

\section{REFERENCIAS BIBLIOGRÁFICAS}

Alovisi, M.; Pasqualini, D.; Musso, E.; Bobbio, E.; Giuliano, C.; Mancino, D.; Scotti, N. \& Berutti, E. Influence of Contracted Endodontic Access on Root Canal Geometry: An in vitro Study. J. Endod., 44(4):614-20, 2018.

Benfica e Silva, J.; Leles, C. R.; Alencar, A. H. G.; Nunes, C. A. B. C. M. \& Mendonça, E. F. Digital subtraction radiography evaluation of the bone repair process of chronic apical periodontitis after root canal treatment. Int. Endod. J., 43(8):673-80, 2010.

Carvalho, F. B.; Gonçalves, M. \& Tanomaru-Filho, M. Evaluation of chronic periapical lesions by digital subtraction radiography by using Adobe Photoshop CS: a technical report. J. Endod., 33(4):493-7, 2007.

Chlup, Z.; Žižka, R.; Kania, J. \& Pr`ibyl, M. Fracture behaviour of teeth with conventional and mini-invasive access cavity designs. J. Eur. Ceram. Soc., 37(14):4423-9, 2017.

D'amico, Y. C.; Neto, U. X da S.; Westphalen, V. P. D.; Carneiro, E. \& Kowalczuck, A. Fracture strength of teeth with access cavity preparation with operating microscope or on buccal surfaces. Braz. Dent. Sci., 22(1):88-93, 2019.

Fan, W.; Fan, B.; Gutmann, J. L. \& Fan, M. Identification of a Cshaped canal system in mandibular second molars. Part III. Anatomic features revealed by digital subtraction radiography. J. Endod., 34(10):1187-90, 2008. 
Fatima, K.; Nair, R.; Khasnis, S.; Vallabhaneni, S. \& Patil, J. D. Efficacy of rotary and reciprocating single-file systems on different access outlines for gutta-percha removal in retreatment: An in vitro study. J. Conserv. Dent., 21(4):354-8 2018.

Gutmann, J. L. Minimally invasive dentistry (Endodontics). J. Conserv. Dent., 16(4):282-3, 2013.

Iqbal, M. K.; Maggiore, F.; Suh, B.; Edwards, K.R.; Kang, J. \& Kim, $\mathrm{S}$. Comparison of apical transportation in four $\mathrm{Ni}-\mathrm{Ti}$ rotary instrumentation techniques. J. Endod., 29(9):587-91, 2003.

Krapez, J. \& Fidler, A. Location and dimensions of access cavity in permanent incisors, canines, and premolars. J. Conserv. Dent., 16(5):404-7, 2013.

Krishan, R.; Paqué, F.; Ossareh, A.; Kishen, A.; Dao, T. \& Friedman, $S$. Impacts of conservative endodontic cavity on root canal instrumentation efficacy and resistance to fracture assessed in incisors, premolars, and molars. J. Endod., 40(8):1160-6, 2014.

Kunert, G. G.; Camargo Fontanella, V. R.; de Moura, A. A. M. \& Barletta, F. B. Analysis of apical root transportation associated with ProTaper Universal F3 and F4 instruments by using digital subtraction radiography. J. Endod., 36(6):1052-5, 2010.

Landis, J. R. \& Koch, G. G. The measurement of observer agreement for categorical data. Biometrics., 33(1):159-74, 1977.

Leoni, G. B.; Versiani, M. A.; Pécora, J. D.; Damião de Sousa-Neto, $M$. Micro-computed tomographic analysis of the root canal morphology of mandibular incisors. J. Endod., 40(5):710-6, 2014.

Lertchirakarn, V.; Palamara, J. E. A. \& Messer, H. H. Patterns of vertical root fracture: factors affecting stress distribution in the root canal. J. Endod., 29(8):523-8, 2003.

Logani, A.; Singh, A.; Singla, M. \& Shah, N. Labial access opening in mandibular anterior teeth--an alternative approach to success. Quintessence Int., 40(7):597-602, 2009.

Mauger, M. J.; Waite, R. M.; Alexander, J. B. \& Schindler, W. G. Ideal endodontic access in mandibular incisors. J. Endod., 25(3):206-7, 1999.

Novy, B. B. \& Fuller, C. E. The material science of minimally invasive esthetic restorations. Compend. Contin. Educ. Dent., 29(6):33846, 2008.

Özyürek, T.; Ülker, Ö.; Demiryürek, E. Ö. \& Yılmaz, F. The Effects of Endodontic Access Cavity Preparation Design on the Fracture Strength of Endodontically Treated Teeth: Traditional Versus Conservative Preparation. J. Endod., 44(5):800-5, 2018.

Plotino, G.; Grande, N. M.; Isufi, A.; Ioppolo, P.; Pedullà, E.; Bedini, R.; Gambarini, G. \& Testarelli, L. Fracture Strength of Endodontically Treated Teeth with Different Access Cavity Designs. J. Endod., 43(6):995-1000, 2017.

Queiroz, P. M.; Nascimento, H. A. R.; da Paz, T. D. J.; Anacleto, F. N. \& Freitas, D. Q. Accuracy of Digital Subtraction Radiography in the Detection of Vertical Root Fractures. J. Endod., 42(6):896-9, 2016.

Reeh, E. S.; Messer, H. H. \& Douglas, W. H. Reduction in tooth stiffness as a result of endodontic and restorative procedures. J. Endod., 15(11):512-6, 1989.

Roperto, R.; Sousa, Y. T.; Dias, T.; Machado, R.; Perreira, R. D.; Leoni, G. B.; Palma-Dibb, R. G.; Rodrigues, M. P.; Soares, C. J.; Teich, S.; Sousa-Neto, M. D. Biomechanical behavior of maxillary premolars with conservative and traditional endodontic cavities. Quintessence Int., 50(5):350-6, 2019.

Rover, G.; Belladonna, F. G.; Bortoluzzi, E. A.; De-Deus, G.; Silva, E. \& Teixeira, C. S. Influence of access cavity design on root canal detection, instrumentation efficacy, and fracture resistance assessed in maxillary molars. J. Endod., 43(10):1657-62, 2017.

Rover, G.; de Lima, C. O.; Belladonna, F. G.; Garcia, L. F. R.; Bortoluzzi, E. A.; Silva, E.; Teixeira, C. S. Influence of minimally invasive endodontic access cavities on root canal shaping and filling ability, pulp chamber cleaning and fracture resistance of extracted human mandibular incisors. Int. Endod. J., 53(11):15309, 2020.
Sabeti, M.; Kazem, M.; Dianat, O.; Bahrololumi, N.; Beglou, A.; Rahimipour, K. \& Dehnavi, F. Impact of Access Cavity Design and Root Canal Taper on Fracture Resistance of Endodontically Treated Teeth: An Ex Vivo Investigation. J. Endod., 44(9):14026, 2018.

Shrout, P. E. \& Fleiss, J. L. Intraclass correlations: uses in assessing rater reliability. Psychol. Bull., 86(2):420-8, 1979.

Sunku, R.; Roopesh, R.; Kancherla, P.; Perumalla, K. K.; Yudhistar, P. V.; Reddy, V. S. Quantitative digital subtraction radiography in the assessment of external apical root resorption induced by orthodontic therapy: a retrospective study. J. Contemp. Dent. Pract., 12(6):422-8, 2011.

Varghese, V. S.; George, J. V.; Mathew, S.; Nagaraja, S.; Indiresha, H. N. \& Madhu, K. S. Cone beam computed tomographic evaluation of two access cavity designs and instrumentation on the thickness of peri-cervical dentin in mandibular anterior teeth. J. Conserv. Dent., 19(5):450-4, 2016.

Yahata, Y.; Masuda, Y. \& Komabayashi, T. Comparison of apical centring ability between incisal-shifted access and traditional lingual access for maxillary anterior teeth. Aust. Endod. J., 43(3):123-8, 2017.

Dirección para correspondencia:

Carlos G. Adorno

Facultad de Odontología

Universidad Nacional de Asunción

Avda. España c/ Calle Brasil

Asunción

PARAGUAY

E-mail: cgadorno@odo.una.py 